sense oligonucleotides in the eye, resulted in impaired axonal growth from retinal neurons and in the reduction of the thickness of the inner plexiform layer.

Although synaptic vesicle exocytosis and recycling occurs throughout the distal arbor of developing axons ${ }^{11}$, axonal elongation is likely to involve constitutive exocytosis at the growth cone of a distinct class of vesicles ${ }^{1}$. So implicating SNAP-25 in axonal elongation is in agreement with its having a function independent of synaptic vesicle exocytosis. It will be of interest to determine whether SNAP-25a is involved in axonal elongation, while SNAP-25b, which is dramatically upregulated during synapse formation, is involved in synaptic vesicle exocytosis.

Does SNAP-25 depletion affect exocytosis from neuronal cell bodies and dendrites? Osen-Sand et al. ${ }^{1}$ do not report any obvious effect of this sort. Yet, in all cells of higher eukaryotes, exocytosis is required not only for growth and secretion, but also for the very active exoendocytotic recycling that takes place at the cell surface. Given that SNAP-25 has a homologue in yeast, SNAP-25 homo- logues are likely to carry out housekeeping functions in all cells; a SNAP-25 isoform related (or identical) to nonneuronal isoforms of SNAP-25 may have this function in the cell body of neurons depleted in SNAP-25.

The characterization of the SNAP-25 gene family, and of the proteins' precise mechanism of action, are the next tasks to be tackled. Given the speed of advance, we should not have to wait long for them to be accomplished.

Pietro De Camilli is in the Department of Cell Biology and the Howard Hughes Medical Institute, Yale University School of Medicine, New Haven, Connecticut 06510, USA.

1. Osen-Sand, A et al Nature 364, 445-448(1993).

2. Oyler, G. A. et al. J. Cell Biol. 109, 3039-3052 (1989)

3. Bark, I. C. J. molec. Biol. (in the press).

4. Hess, D. T., Slater, T. M., Wilson, M. C. \& Pate Skene, J. H. J. Neurosci. 12, 4634-4641 (1992)

5. Oyler, G. A., Polli, J. W., Wilson, M. C. \& Billingsley, M. L. Proc. natn. Acad. Sci. U.S.A. 88, 5247-5251 (1991)

6. Liu, Y. etal. Neuron 6, 411-420(1991)

7. Christgau, S. etal. J. Cell Biol. 118, 309 (1992).

8. Söllner, T. et al. Nature 362, 318-323 (1993).

9. Schiavo, G. etal. Nature 359, 832-835 (1992)

10. Link, E. et al. Biochem. biophys. Res. Commun. 189 1017-1023 (1992)

11. Matteoli, M., Takei, K., Perin, M. S., Südhof, T. C. \& De Camilii, P. J. Cell Biol. 117, 849-861(1992).

\title{
Next swing of the pendulum
}

\section{Charles D. Bailyn}

IN the middle of the 1970 s, when X-ray sources were discovered in globular star clusters, there was considerable excitement surrounding the possibility that these sources might contain black holes. Ten years later, it was generally agreed that this interpretation was false. Now, in a new swing of the pendulum, two papers on pages 421 and 423 of this issue $e^{1.2}$ suggest that globular clusters may indeed contain black holes, albeit of much smaller mass than those envisaged in the $1970 \mathrm{~s}$.

The original suggestion was that the cluster X-ray sources could be explained by a black hole several hundred times the mass of the Sun, sitting in the cluster centre accreting gas and stars from its dense surroundings. Theoretical studies of cluster evolution which indicated that the core of a cluster should collapse to infinite density in a finite time lent credence to this idea. But the X-ray sources could also be explained as binary star systems in which a neutron star accreted material from its companion. Supporting this hypothesis was the clear evidence of binarity in several similar X-ray sources located outside clusters. The significantly greater frequency of X-ray sources in clusters compared with the rest of the galaxy was explained as a consequence of 'tidal capture', a process in which stars in a dense cluster transfer sufficient kinetic energy into tidal oscillations to

\section{create tight binary systems.}

Two lines of evidence showed the latter hypothesis to be preferable. First, the mysterious X-ray bursts observed in the cluster sources were convincingly modelled as thermonuclear explosions of material accreted onto the surface of a neutron $\mathrm{star}^{3}$. Accretion onto black holes would not create densities high enough to initiate the bursts, as the holes lack a surface on which material can accumulate. Secondly, X-ray observations with high spatial resolution demonstrated that the X-ray sources were not coincident with the cluster centre ${ }^{4}$, as would be expected of black holes significantly more massive than an individual star. Indeed, the spatial distribution of the sources was found to be compatible with binary systems of two solar masses, as predicted by the neutron star model. In the face of this contrary evidence, the initial enthusiasm for black holes diminished greatly.

Interest in stellar-sized black holes in general has been heightened recently by both observational and theoretical developments. Observationally, it has been shown that a number of transient X-ray sources outside globular clusters contain compact objects of greater than three times the mass of the Sun ${ }^{5-7}$. Basic considerations of general relativity imply that these objects must be black holes. The boom in supernova modelling following the observation of the supernova in the Large Magellanic Cloud in 1987 and the success of supernova search programmes has reinforced the idea that some supernova explosions can leave behind black holes $^{8-9}$. Extrapolations from these results has led to an estimate of a population of over a hundred million stellar-sized black holes in our Galaxy alone ${ }^{10}$.

Now, Kulkarni and his collaborators ${ }^{1}$ and Sigurdsson and Hernquist ${ }^{2}$ have explored the consequences of the less massive black holes in globular clusters. Both groups demonstrate that the holes, being more massive than a typical cluster member, will rapidly sink to the centre of the cluster, where they will interact with each other. These interactions are likely to result in the expulsion from the cluster of most if not all of the holes on a relatively short timescale. If a hole or two remains behind, however, there may be interesting observational consequences, particularly if, as seems likely, they form binary pairs.

Kulkarni and collaborators suggest that binaries comprising a black hole and a normal star may result in a transient $\mathrm{X}$-ray-emitting system, just as they do outside clusters. Sigurdsson and Hernquist point out that binary systems containing two black holes may have a devastating effect on the surrounding normal stars, stripping material from them, and in some cases disrupting them completely. These effects may, they say, account for recently observed anomalies in the stellar populations at the centres of the densest clusters ${ }^{11}$.

Thus we have come full circle since the 1970s. At that time, unexplained observations prompted the theoretical speculation that globular clusters might contain black holes. Now, theoretical results are suggesting observational tests of the presence of a rather different black hole population. Direct or indirect observational confirmation would give us a fascinating new window on the complex problems of the evolution of globular clusters, as well as on the origin of the black holes themselves.

Charles Bailyn is in the Department of Astronomy, Yale University, New Haven, Connecticut 06511, USA.

\footnotetext{
Kulkarni, S. R., Hut, P. \& McMillan, S. Nature 364 421-423(1993).

2. Sigurdsson. S. \& Hernquist, L. Nature 364, 423- 425 (1993)

3. Ayasli, A. \& Joss, P. C. Astrophys. J. 256, 637-665 (1982).

4. Grindlay, J. E. et al Astrophys J. 282, L13-L16 (1984)

5. McClintock, J. E. \& Remillard, R. A. Astrophys. J. 308, 110-122(1986).

6. Casares, J., Charles, P. A. \& Naylor, T. Nature 355 614-617 (1992).

7. Remiliard, R. A., McClintock, J. E. \& Bailyn, C. D. Astrophys. J. 399, L145-L149 (1992).

8. Woolsey, S. E. \& Weaver, T. A. A. Rev. Astr. Astrophys. 24 205-254 (1986).

9. Maeder. A. Astr. Astrophys. 264, 105-120 (1992)

10. van den Heuvel, E. P. J. in Proc. Satellite Symp. 3: Space Sciences with Particular Emphasis on High-Energy Astrophysics 29-36 (ESA, 1992)

11. Djorgovski, S. et al. Astrophys. J. 372, L41-L44 (1991)
} 